\title{
A new online monitoring and management system for accidental pollution events developed for the regional water basin in Ningbo, China
}

\author{
Gaosheng Zhang, Linlin Chen, Yuedan Liu, TaeSoo Chon, Zongming Ren, \\ Zijian Wang, Jianping Zhao and Yangyong Zhao
}

\begin{abstract}
$\overline{\text { ABSTRACT }}$
Due to urgency of the accidental pollution events (APE) on one side and the variability in water quality data on the other side, a new online monitoring and management system (OMMS) was developed for the purpose of sustainable water quality management and human health protection as well. The Biological Early Warning System (BEWS) based on the behavioral responses (behavior strength) of medaka (Oryzias latipes) were built in combination with the physico-chemical factor monitoring system (PFMS) in OMMS. OMMS included a monitoring center and six monitoring stations. Communication between the center and the peripheral stations was conducted by the General Packet Radio Service (GPRS) network transmission complemented by a dial-up connection for use when GPRS was unavailable. OMMS could monitor water quality continuously for at least 30 days. Once APES occurred, OMMS would promptly notify the administrator to make some follow up decisions based on the Emergency Treatment of APE. Meanwhile, complex behavioral data were analyzed by Self-Organizing Map to properly classify behavior response data before and after contamination. By utilizing BEWS, PFMS and the modern data transmission in combination, OMMS was efficient in monitoring the water quality more realistically.

Key words | accidental pollution events, online monitoring and management system, water quality monitoring

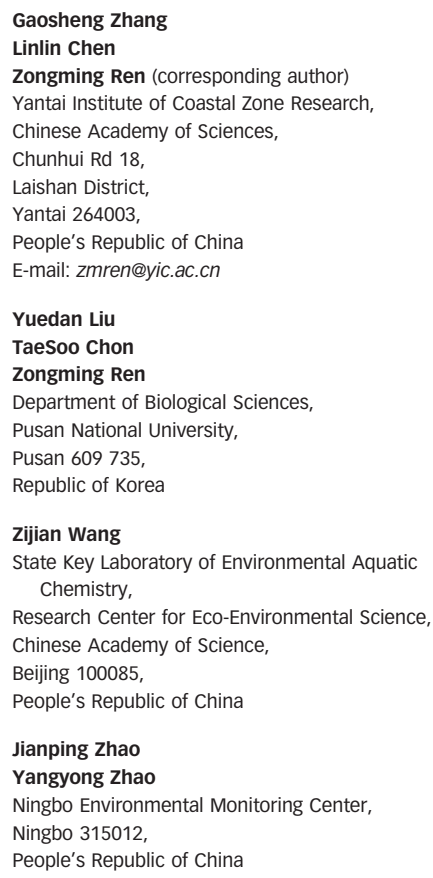

\section{INTRODUCTION}

The extensive application of pesticides and the frequent transport of chemicals have been potential threats to human health and the ecological balance of aquatic ecosystems worldwide (Lyons et al. 2002). Indeed, accidental pollution events (APE) often occurred (Bridges 2003; Zhang et al. 20Io), and identification of water pollutants and polluters was sometimes even not possible (Bode \& Nusch 1999; Ren \& Wang 2010). Due to APE and variability in water quality data (Table 1) (Zhang et al. 2006), it is therefore urgent for the administrators to strengthen the capability of online monitoring and proper judgment.

The development of methods on water quality management has been a consequence of the modernization process. Numerous reports have appeared during the last
15 years (Richards et al. 1996; Davies \& Mazumder 2003; Vanderzalm et al. 20II). In China, for instance, the Chinese Government has proposed numerous protocols and measuring systems for aquatic environment management. In the seven major river basins, more than 100 monitoring stations have been built (RDDS 20I0). These stations are equipped according to the National Environmental Monitoring Station Construction Standards (NEMSCS 2007). However, the research data and measuring equipment are still insufficient in detecting unexpected APEs. It is indeed difficult to confirm abrupt accidents, since no system in these stations is capable of alarming the sudden change of water quality, especially in the case of combined pollution. In addition, correct estimation of pollution levels is difficult, once APE 
Table 1 | Statistics of aquatic pollution events in China from 1985 to 2005

Cause of accidents

Number

Production and application of dangerous chemicals along 58 rivers and lakes

Pesticides

Traffic accidents

Spreading poison with intent

Other reasons for accidents

Uncertainty of pollutants

Total

102

has been detected. Different chemicals may have different toxic effects on organisms. Physico-chemical factor (PF) monitoring alone may not be sufficient to measure pollution levels properly. Supportive measurements may be also required for more realistic monitoring of APE. Biological monitoring is an alternative to supportive measures.

Some efforts have been made in recent years to develop online biomonitoring systems for detecting APE: The Fluorescence System (Rodriguez et al. 2002), the DaphniaToximeter (Green et al. 2003), and the Biological Early Warning System (BEWS) (Ren \& Wang 20IO). In this study, we propose a new online monitoring system to combine physico-chemical and behavioral data to realize the online monitoring and management of APEs with SelfOrganizing Map (SOM) analysis.

\section{METHODS}

\section{Background information of Ningbo Water Basin (NWB)}

Ningbo serves as one of the chemical industry bases in east China. In recent years, Ningbo Municipality has carried out effective measures by forbidding fishing, swimming and the direct discharge of sewage and garbage in most of the rivers and reservoirs. These measures somewhat effectively controlled pollutant sources. However, the industrial and agricultural production along rivers and reservoirs would contribute to sudden occurrence of APE. Transportation of chemicals may also be a great threat to water supply in Ningbo (Figure 1). According to Zhang et al. (2006), about $39 \%$ of all APEs in China were caused by traffic accidents (Table 1).

At present, six monitoring stations with a monitoring center have been established (Figure 1). The stations CS, $\mathrm{XS}$, BRS and FS were built for the purpose of source

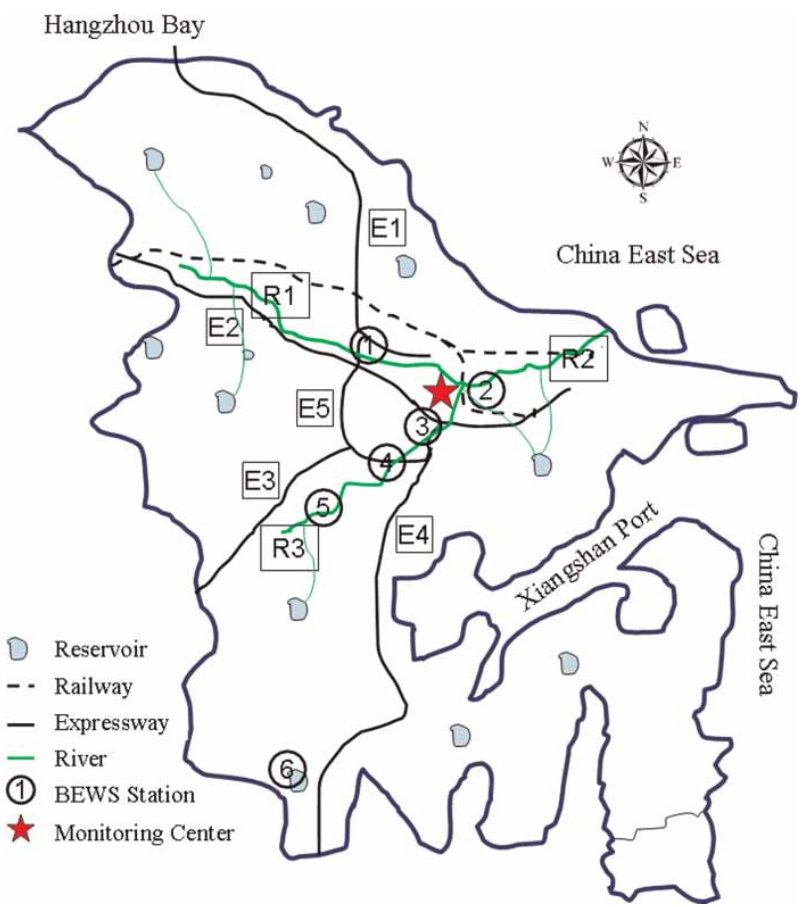

Figure 1 | Schematic diagram of NWB. (1) the Chengshandu Station (CS), (2) the Sewage Treatment Plant Station (STPS), (3) the Younger Corporation Station (YCS) for the Younger Corporation which is a garment manufacturing company (4) the Fangqiao Station (FS), (5) the Xiaozhen Station (XS), (6) the Baixi Reservoir Station (BRS), R1 the Yuyaojiang River, R2 the Yongjiang River, R3 the Fenghuajiang River, E1 the Hangzhou Bay Expressway, E2 the Hangyong Expressway, E3 the Yongjin Expressway, E4 the Yongtaiwen Expressway, E5 the Ningbo Belt Expressway, and the Hangyong Railway. The monitoring center was in the Ningbo Environmental Monitoring Center.

water security, while YCS and STPS were constructed to protect NWB from pollution due to direct discharge of sewage. These stations are controlled by an automatic alarm system for hydrological data acquisition and transmission. Communication between the stations and the center is mainly conducted by the general packet radio service (GPRS) network transmission, being complemented with a dialup connection for use when GPRS is unavailable.

\section{BEWS}

In order to conduct online biomonitoring of APE in China, BEWS was developed by the Chinese Academy of Sciences for six years (Ren \& Wang 20Io). In BEWS, test organisms were placed in a flow-through test chamber (different sizes for different aquatic organisms), which was closed off on both sides with a silicone net (pore diameter was about $0.5 \mathrm{~mm}$ ). Each BEWS had eight test chambers. The behavior signal was presented by behavior strength (BS) sampled automatically by BEWS every second (Ren \& Wang 20ro). 
Average values in every 6 min were produced as output and were used to present behavioral changes in this study.

Medaka (Oryzias latipes) being proposed as the standard test fish (OECD I999), was used as the indicator species. An online biomonitoring of water quality in Jingmi Diversion Canal for 30 days suggested that medaka could live without feeding for at least 25 days and the stability of BEWS was acceptable for online monitoring (Ren et al. 2009).

\section{Physico-chemical Factor Monitoring System (PFMS)}

For effective environmental management of NWB, a new Online Monitoring and Management System (OMMS) is proposed to provide reliable detection of APEs. The system includes three parts: the first part is combined use of behavioral assessment (BEWS) and environmental measurement with PFMS, the second part is utilization of SOM for analyzing complex behavioral data, and the third part is operation of a real time environmental management network.

PFMS was built based on the different PF detectors, which included temperature (T), turbidity (TUB), $\mathrm{pH}$, dissolved oxygen (DO), electric conductivity (EC), chemical oxygen demand (COD), ammonia (NH-N), total phosphorus (TP), and total nitrogen (TN). According to previous research on the effects of $\mathrm{PF}$ on the behavioral responses of aquatic organisms (Ren 2008) and GB3838-2002 (2002), the normal PF range could be specified as $10-30{ }^{\circ} \mathrm{C}(\mathrm{T})$, $\leq 20$ NTU (TUB), 6-9 (pH), $\geq 2 \mathrm{mg} / \mathrm{L}$ (DO), $\leq 40 \mathrm{mg} / \mathrm{L}$ (COD), $\leq 2.0 \mathrm{mg} / \mathrm{L}(\mathrm{NH}-\mathrm{N}), \leq 0.2 \mathrm{mg} / \mathrm{L}(\mathrm{TP})$, and $\leq 2.0 \mathrm{mg} /$ L (TN). PF may unexpectively affect the behavioral responses of aquatic organisms and thus would produce false results (Ren 2008). Therefore, these factors were taken into account when the optimal allocation of source water was decided.

The operation of BEWS alone may not satisfy the requirements for online monitoring and management of accidental events in NWB. Therefore, a new OMMS for NWB was developed by combining PFMS and BEWS under the scheme of an automatic alarm system.

\section{SOM}

SOM performs a non-linear projection of data onto a space in two dimensions and provides a comprehensive map of input data by unsupervised learning (Kohonen 200I). The size of the SOM was determined heuristically in such a way that variations of input data would be comprehensible to the reader. In this way, the highest variance in the input data will be projected along with the vertical axis while the following variance would be accordingly presented on the horizontal axis. Optimal size was adjusted to $14 \times 10$ nodes based on degree of discrimination among the grouped nodes after training.

The Euclidian distance $\left(d_{j}(t)\right)$ at the $j$-th node on the SOM between weight at iteration time $t$ and the input vector was calculated through learning processes:

$d_{j}(t)=\sum_{i=0}^{P-1}\left[x_{i}-w_{i j}(t)\right]^{2}$

where $x_{i}$ is the value of the $i$-th parameter, $w_{i j}(t)$ is the weight between $i$-th parameter and the $j$-th node on the SOM and $P$ is the number of the parameter. In this study parameters were represented by T, TUB, pH, DO, COD, NH-N, TP, EC, TN chlorine and chlorophyll a.

The best matching neuron, which has the minimum distance, was chosen as the winner. For the best matching neuron and its neighborhood neurons, the new weight vectors are updated as:

$w_{i j}(t+1)=w_{i j}(t)+a(t)\left[x(t)-w_{i j}(t)\right]$

where $t$ is the iteration time and $a(t)$ is the learning rate.

As the input data were fed to the SOM for training (Equation (2)), the weights of the best matching unit and computation nodes close to it were adjusted towards the input vector through interactive calculation. In order to reveal the degree of association between the SOM units, the Ward's linkage method was used to cluster the movement data based on the dendrogram according to the Euclidean distance (Ward I963). The learning process of the SOM was conducted using the SOM Toolbox by following optimization in algorithm (Vesanto et al. 200o). A detailed description of the SOM of behavioral data can be referred to Liu et al. (2OII).

\section{$\overline{\text { RESULTS AND DISCUSSION }}$}

\section{Integrated operation of OMMS}

As shown in Figure 2, the alarm output by OMMS is determined by the results from BEWS and PFMS. In the absence of any unusual behavioral responses by the test organisms in BEWS, the water status would be considered normal although one or a few components in the PF are still out 


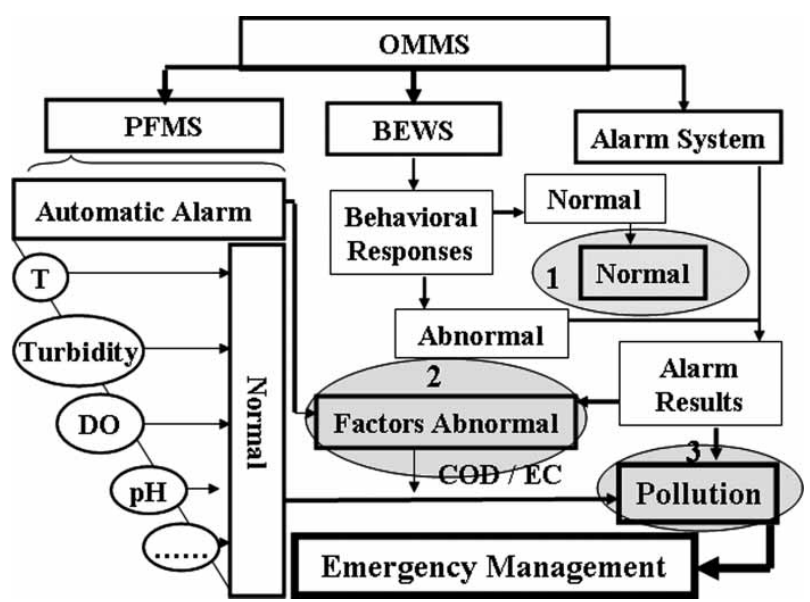

Figure 2 Technique design pattern of the new OMMS for NWB. The alarm output 1 (Normal) meant that the result of BEWS was normal, and the results of PFMS were not considered; the alarm output 2 (Factors Abnormal) meant that the result of BEWS was abnormal, if a few factors in PFMS were abnormal too, the alarm system would begin to process which PFs were responsible for the results. Once it was $\mathrm{COD}$ and $\mathrm{EC}$, or there was no PF abnormal, the alarm output would be 3 (Pollution), which suggested that APE might occur.

of the acceptable range. In order to cope with this type false alarm, the step-by-step approach was applied to the combination of PFMS and BEWS. Considering that behavioral response presents the overall state of the water quality and that each PF shows locally one aspect of water quality, behavioral responses in BEWS were first checked. Once the behavioral responses of the test organisms in BEWS suggested that there was something abnormal due to an abrupt change, the alarm system would begin to process which PFs were responsible for the abrupt difference in BEWS. In this way, APEs could be more reliably detected.

OMMS in the monitoring center could be connected to the Internet server (http://124.89.192.132/demo/) with direct access permission (Authorization Code), with the status of each monitoring station being indicated online. Based on the information provided by the OMMS network, optimal allocation of water in NWB and water supply could be determined accordingly. Once an APE was detected, some emergency treatment would be carried out, which included stopping the source water supply from one place and starting from another to realize the optimal allocation of water in NWB, emergency detection of water polluter(s) and concentration(s), and emergency treatment of the APE.

\section{Environmental management of NWB}

Figure 3 shows the changes in BEWS with medaka along with other factors in PFMS of XS (on August 9, 2009) displayed by the view software of BEWS. The sudden decrease in the curve (vertical box in Figure 3) suggests

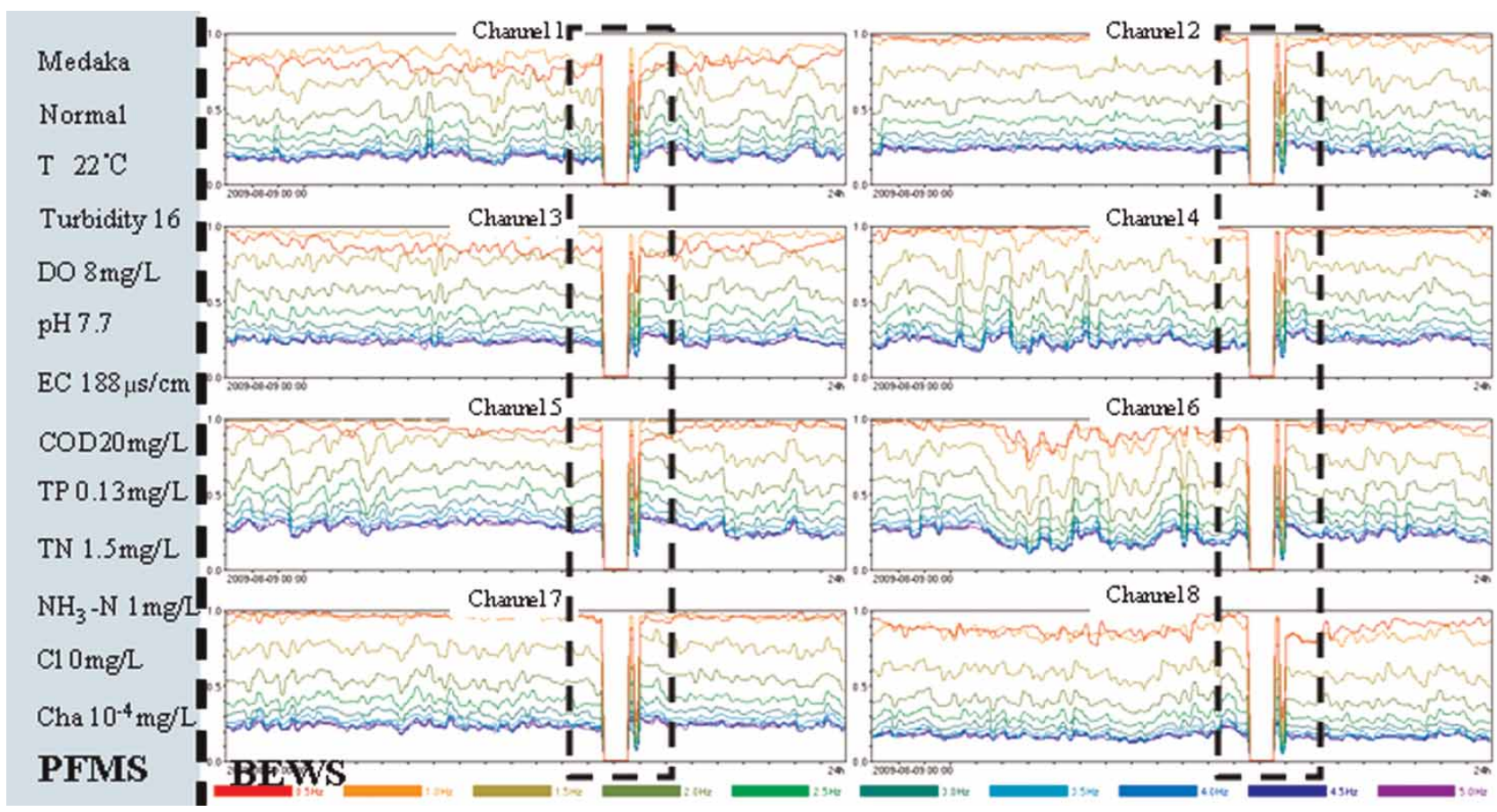

Figure 3 | The monitoring results of XS in August 9, 2009. The left part showed the results of PFMS at the end of monitoring and the right part showed the results of BEWS. The box showed the phase of the behavior signal as abnormal. 
that there was an abrupt impact on water (decreased from about 0.9 to 0 in $10 \mathrm{~min}$ ). COD correspondingly increased from 20 to $40 \mathrm{mg} / \mathrm{L}$ and EC also increased from $200 \mu \mathrm{s} /$ $\mathrm{cm}$ to more than $1,500 \mu \mathrm{s} / \mathrm{cm}$ suddenly at this time. Along with change in BEWS values and the help of the technique design pattern showed in Figure 2, OMMS gave a judgment that an accidental event may occur (Onsager ig68; Hartnett et al. 1998). Based on the Emergency Treatment of APE in NWB, some decisions could be made as follows:

1 st, stop the source water supply from Xiaozhen intake, and increase the water supply from CS, BRS and FS.

2nd, emergency detection of water polluter(s) and concentration(s).

3rd, emergency treatment of the APE.

The BS values were classified by using the SOM with PF measured from each unit time (6 min). Figure 4(a) shows the clustered SOM based on the clustering dendrogram (Figure 4 (b)). Four patterns were identified according to the Ward linkage clustering (Figure 4(b)) (Ward I963). The relatively high values of BS similarly showed in the clusters I, II and III, while the lower values of BS were presented in the cluster IV. Figure 4(c) showed the BS change in clusters according to the SOM reorganization. The behavioral data matching to cluster IV were low for 882-936 min and BS increased to a higher level in different clusters accordingly.

The profiles of PF can also be visualized according to the location on the SOM (Figure 4(d)). Cluster I on the upper right area of the map (Figure 4(a)), for instance, was mainly characterized by high TP, low TN and low NH-N. Cluster II was observed at the mid-upper left area of the map and was presented with relatively low temperature, high DO and low COD. Cluster III on the bottom left showed the highest levels of TN and NH-N. In contrast, the highest $\mathrm{T}$ and TUB, the maximum COD and EC, and the lower $\mathrm{pH}$ were presented in cluster IV on the bottom right. Cluster IV indeed matched abnormal ranges in environmental factors. The accidental event might be combined pollution due to the association between COD and
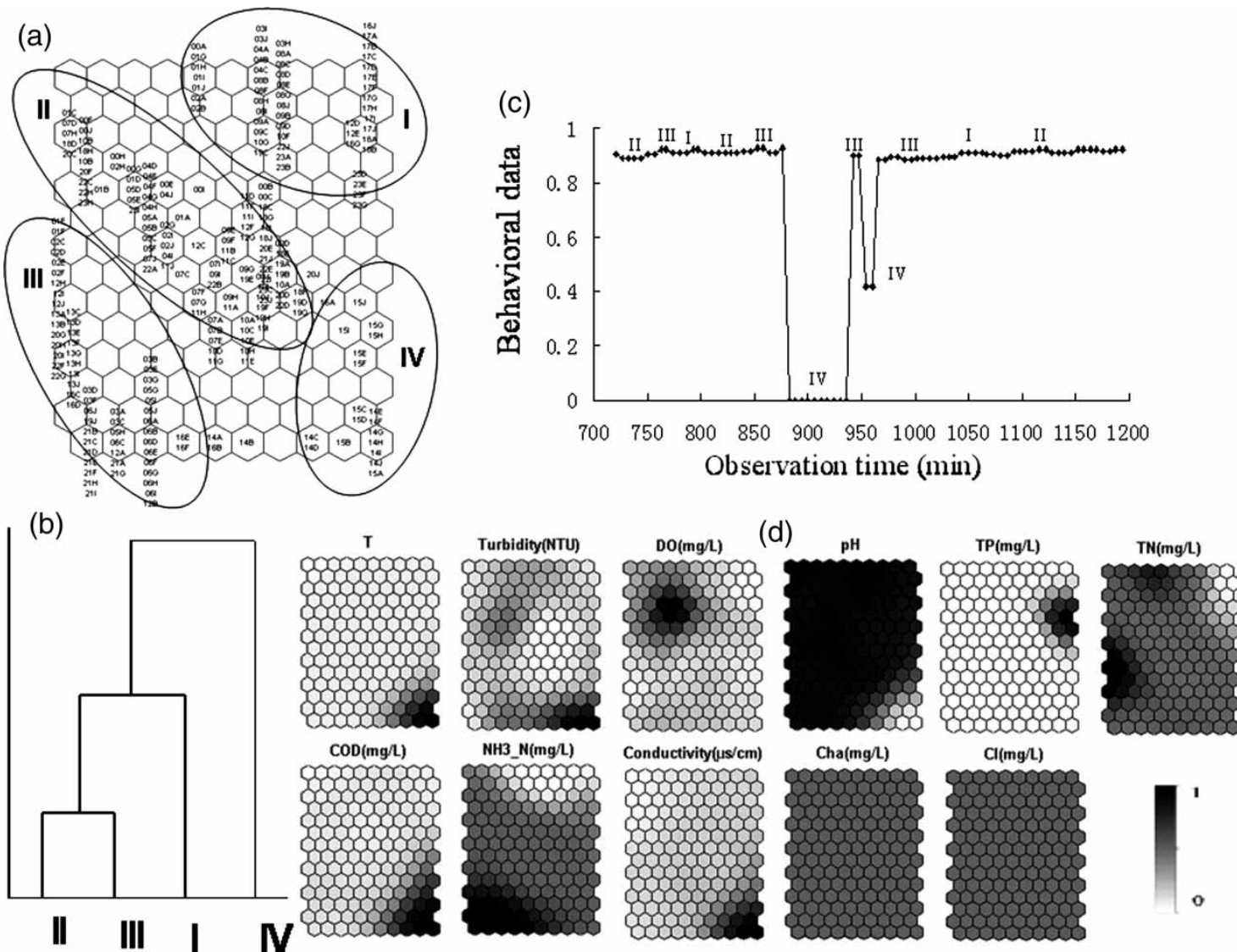

Figure 4 | The map trained by the SOM for pattering behaviors. (a) Four clusters classified by the SOM (the characters in the hexagon indicate the behavioral signal in each 6 min), (b) cluster distances according to the Ward's linkage method, (c) shows the dynamic changes of mean value of the abnormal behavioral data (882-936 min) based on the som analysis and (d) the profile of PF matching to clusters based on the trained SOM. The values in the vertical bar in the top row indicate normalized parameters. 
Total Organic Carbon (TOC) (Hartnett et al. 1998) and the relationship between EC and ion concentration (Onsager I968).

Meanwhile, some test medaka revived after about $1.5 \mathrm{~h}$. This indicated that recovery may have occurred to some degree. At last, this incident matched with field data proved that it was overflow of sewage with divalent cadmium $\left(\mathrm{Cd}^{2+}\right)$, as a byproduct of an Electroplate Factory running upstream. The concentration of $\mathrm{Cd}^{2+}$ was about 1 $\mathrm{mg} / \mathrm{L}$ at this time.

Since the event was transient pollution, the source water supply from Xiaozhen intake started again after the pollutants spread to the downstream. However, as no better solution could deal with the $\mathrm{Cd}^{2+}$ pollution in the larger scope, environmental staff were requested to continuously detect the water quality along the Fenghuajiang River and the Yongjiang River until the $\mathrm{Cd}^{2+}$ concentration satisfied the requirements of GB3838-2002 (2002) $(<0.01$ $\mathrm{mg} / \mathrm{L})$.

\section{CONCLUSIONS}

To satisfy the requirements of APE online monitoring, this paper has proposed an OMMS developed for NWB, by including combined behavior and environmental measurements, SOM analysis and an environmental management network (Monitoring Center). OMMS could achieve optimal allocation of source water in the regional water basin following APE. The decision for the warning system was accordingly determined by comparing criteria of PF and BS. In this way, complex situations (e.g. the abnormal range in a few PFs matching with abnormal BS) were efficiently identified to draw more realistic alarm. The proposed OMMS provides a realistic means for water quality assessment and management.

In future, the in-situ biological monitoring technique including biomarkers and biosensor could be further developed based on this type of integrative online system.

\section{ACKNOWLEDGEMENTS}

This study was financially supported by the 2011 Post-Doc. Development Program of Pusan National University and China National Key Program for Water Pollution Control (2009 ZX07527-002).

\section{REFERENCES}

Bode, H. \& Nusch, E. A. I999 Advanced river quality monitoring in the RUHR Basin. Water Science and Technology 140 (10), $145-152$.

Bridges, O. 2003 Double trouble: health risks of accidental sewage release. Chemosphere 52, 1373-1379.

Davies, J. M. \& Mazumder, A. 2003 Health and environmental policy issues in Canada: the role of watershed management in sustaining clean drinking water quality at surface sources. Journal of Environmental Management 68, 273-286.

GB3838-2002 2002 Environmental Quality Standard for Surface Water. Environmental Science, China.

Green, U., Kremer, J. H., Zillmer, M. \& Moldaenke, C. 2003 Detection of chemical threat agents in drinking water by an early warning real-time biomonitor. Environmental Toxicology 18 (6), 368-374.

Hartnett, H. E., Keil, R. G., Hedges, J. I. \& Devol, A. H. 1998 Influence of oxygen exposure time on organic carbon preservation in continental margin sediments. Nature 391, 572-575.

Kohonen, T. 200I Self-Organizing Maps, 3rd extended edition. Springer, Berlin.

Liu, Y., Lee, S. \& Chon, T. 2orr Analysis of behavioral changes of zebrafish (Danio rerio) in response to formaldehyde using Self-organizing map and a hidden Markov model. Ecological Modelling 222, 2191-2201.

Lyons, R. A., Wright, D., Fielder, A., McCabe, M., Gunneberg, A. \& Nash, P. 2002 Investigation of an acute chemical exposure incident to fluorinated hydrocarbons. Occupational and Environmental Medicine 57, 577-581.

NEMSCS 2007 The National Environmental Monitoring Station Construction Standards. Ministry of Environmental Protection of China.

OECD (Organization for Economic Co-operation and Development) I999 Final Report of the Fish Expert Consultation Meeting, London, UK, October, 28-29, 1998; Paris, Cedex 16, France.

Onsager, L. 1968 Nobel Lecture - The motion of ions: principles and concepts. Nobel Foundation, Stockholm.

RDDS 2010 Real-time data dissemination system of national surface water quality automatic monitoring. China National Environmental Monitoring Centre. Available from: http://58. 68.130.147/ (accessed 20 April 2011).

Ren, Z. 2008 The Studies on the On-line Biological Early Warning of Water Quality Based on the Behavioral Responses of Aquatic Organisms. PhD Thesis, Chinese Academy of Sciences, Beijing, China.

Ren, Z. \& Wang, Z. 2oIo The differences in the behavior characteristics between Daphnia magna and Japanese Madaka in an on-line biomonitoring system. Journal of Environmental Sciences 22, 703-708.

Ren, Z., Wang, T. \& Zhao, L. 2009 Simulation of early warning and application of on-line biological early warning system for water quality safety. Water Technology 3, 1-3.

Richards, K., Sharp, M. \& Arnold, N. 1996 An integrated approach to modelling hydrology and water quality in glacierized catchments. Hydrological Processes 10 (44), 479-508. 
Rodriguez, M., Sanders, C. A. \& Greenbaum, E. 2002 Biosensors for rapid monitoring of primary-source drinking water using naturally occurring photosynthesis. Biosensors and Bioelectronics 17, 843-849.

Vanderzalm, J. L., Page, D. W. \& Dillon, P. J. 20II Application of a risk management framework to a drinking water supply augmented by stormwater recharge. Water Science and Technology 63 (4), 719-726.

Vesanto, J., Himberg, J., Alhoniemi, E., Parhankangas, J., Team, S. \& Oy, L. 2000 Som Toolbox for Matlab. Techn. Ber., Helsinki University of Technology.
Ward Jr., J. I963 Hierarchical grouping to optimize an objective function. Journal of the American Statistical Association 58, 236-244.

Zhang, W., Lin, X. \& Su, X. 20Io Transport and fate modeling of nitrobenzene in groundwater after the Songhua River pollution accident. Journal of Environmental Management 91, 2378-2384.

Zhang, Y., Wang, D. \& Yang, K. 2006 Statistical analysis on water pollution incident in urban water supply area in China during the year 1985 to 2005. Journal of Safety and Environment 6, 79-84.

First received 29 April 2011; accepted in revised form 23 June 2011 